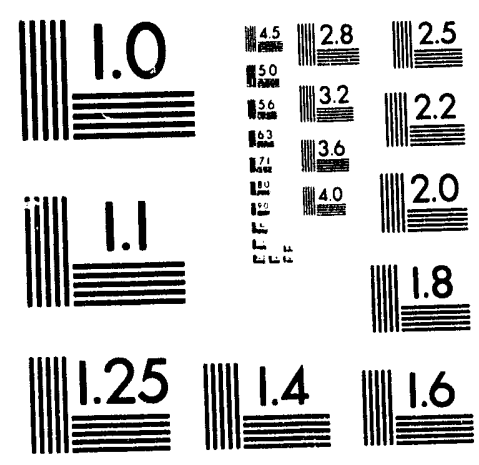



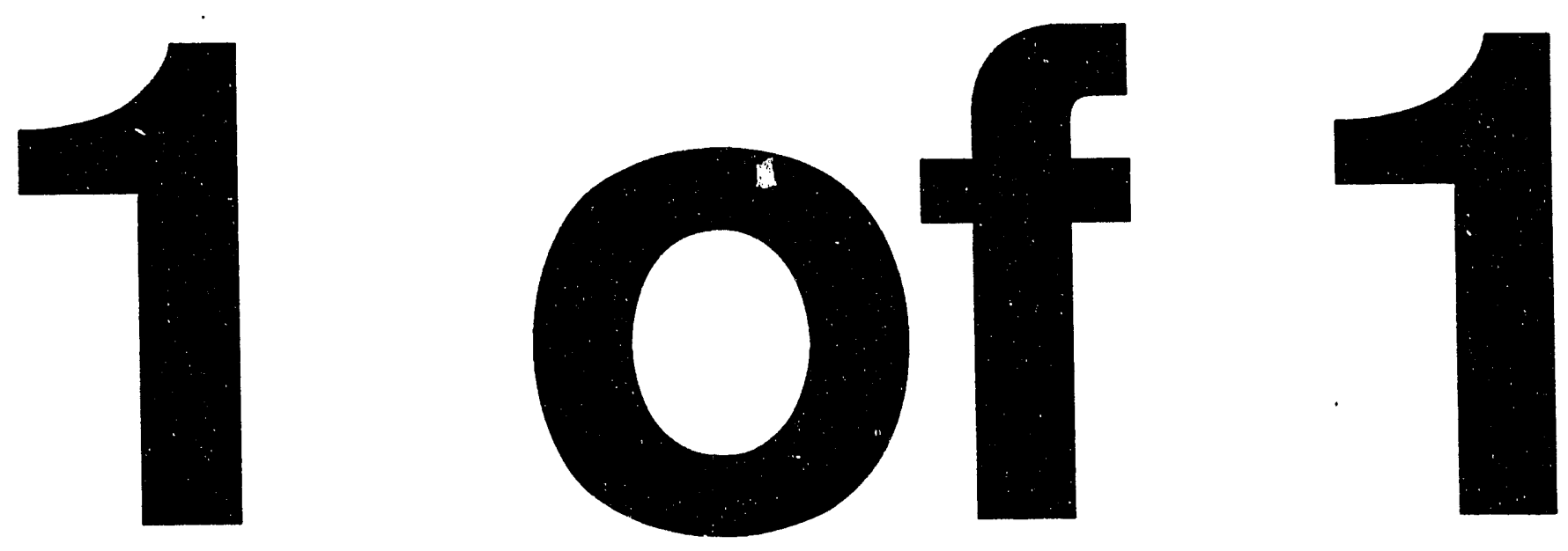


\title{
A Closed-Loop Photon Beam Control Study for the Advanced Light Source*
}

\author{
G. Portmann and J. Bengtsson \\ Lawrence Berkeley Laboratory, University of California \\ 1 Cyclotron Road, Berkeley, CA 94720 USA
}

\begin{abstract}
The third generation Advanced Light Source (ALS) will produce extremely bright photon beams using undulators and wigglers. In order to position the photon beams accurate to the micron level, a closed-loop feedback system is being developed. Using photon position monitors and dipole corrector magnets, a closed-loop system can automatically compensate for modeling uncertainties and exogenous disturbances. The following paper will present a dynamics model for the perturbations of the closed orbit of the electron beam in the ALS storage ring including the vacuum chamber magnetic field penetration effects. Using this reference model, two closed-loop feedback algorithms will be compared -- a classical PI controller and a two degree-offreedom approach. The two degree-of-freedom method provides superior disturbance rejection while maintaining the desired performance goals. Both methods will address the need to gain schedule the controller due to the time varying dynamics introduced by changing field strengths when scanning the insertion devices.
\end{abstract}

\section{INTRODUCTION}

The ALS is designed to store a $400 \mathrm{~mA}, 1.5 \mathrm{GeV}$, multi-bunch electron beam with a lifetime of 6-8 hours. The natural rms emittance is $3.4 \times 10^{-9} \mathrm{~m}$-rad with an estimated rms beam size at the center of the insertion device of $195 \mu \mathrm{m}$ horizontally and $37.4 \mu \mathrm{m}$ vertically. The lattice is based on a cell with a triple bend achromat, repeating twelve times. This leaves room for a maximum of ten insertion devices. The storage ring is optimized to produce photon beams in the VUV to soft X-ray spectral region.

In order to position the photon beams accurate to the micron level, a closed-loop feedback system on the electron beam is being developed. Potential error sources driving the electron beam include: environmental vibrations, magnetic field changes associated with insertion device scanning, magnet power supply ripple, and temperature drift. Steering of the photon beam is done by monitoring the position at two locations and correcting the position and angle of the electron beam through the insertion device. This is done by local correction using four dipole corrector magnets in each plane. The following paper integrates accelerator beam dynamics with feedback control theory for systematic controller design.

-This work was aupported by the Director, Office of Energy Research, Office of Basic Energy Sciences, Material Sciences Division, of the U.S. Department of Energy under the U.S. DOE Contract No. DE-AC03-76SF00098.

MASTER

\section{ALS MAGNET LATTICE}

In order to design a feedback control system for photon beam steering, one must have an accurate closed orbit beam dynamics model. Fig. 1 shows the magnetic lattice for two of the twelve sectors. Each sector is identical except for the type of insertion device. Using the two dipole corrector magnets on either side of the insertion device, the goal of the local bump system is to accurately steer the electron beam though the insertion device without perturbing the bsam position outside the four magnet bunp. This algoritbm must account for focusing changes in the quadrupsles located between the corrector magnets and the field changes of the insertion devices during scanning.

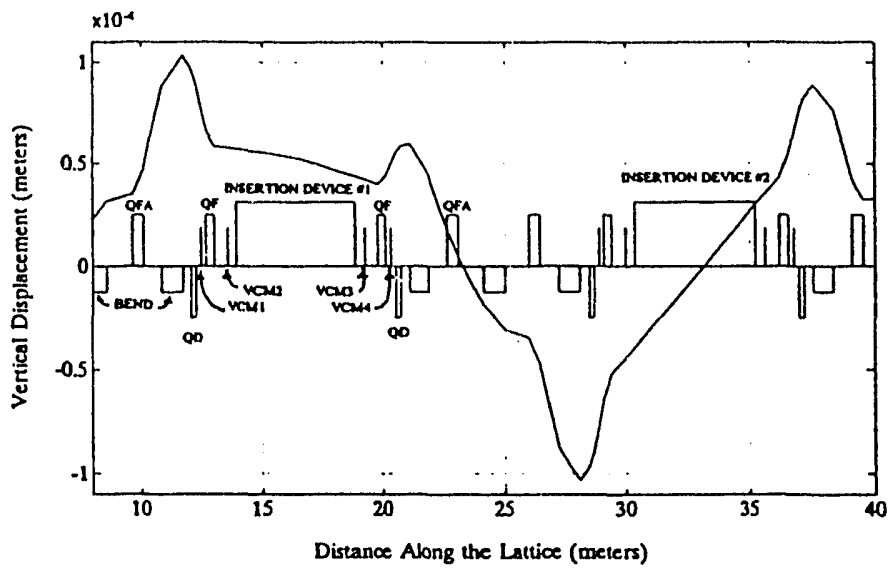

Fig. 1. Closed Orbit for a $10 \mu$-radian dipole kick at VCM1

Since only small perturbations about the equilibrium orbit are being considered, linear moduls are sufficient. If necessary, one can easily generalize to the nonlinear case by using automatic differentiation, [9].

The change of the phase space coordinates $\bar{x}=\left(x, p_{x}\right)$ between two points in the lattice is given by the transport matrix,

$$
\bar{x}_{j}=M_{i \rightarrow j} \bar{x}_{i} .
$$

Therefore, $M_{j \rightarrow j}$ is the one-turn matrix. Consider the closed orbit distortions for a single dipole kick, $\Delta \bar{x}_{i}=\left(0, \Delta \Theta_{2}\right)$. The distortion at location $i$ is then given by

$$
\Delta \overline{\mathrm{x}}_{\mathrm{i}}^{\infty \mathrm{d}}=\left[\mathrm{I}-\mathrm{M}_{\mathrm{j} \rightarrow \mathrm{j}}\right]^{-1} \Delta \overline{\mathrm{x}}_{\mathrm{i}} \text {. }
$$

The distortions at other locations in the lattice can be computed from (1). Fig. 1 shows the distortions of the vertical closed orbit for a .01 milliradian kick from corrector magnet VCM1. By superposition, one can find the contributions to the closed orbit distortions from the four 
corrector magnets to the center of the insertion device and VCM4. Introducing, $\Delta \bar{\Theta}=\left(\Delta \Theta_{1}, \Delta \Theta_{2}, \Delta \Theta_{3}, \Delta \Theta_{4}\right)$, one obtains the following system of equations

$$
\left[\begin{array}{c}
\Delta \overline{\mathrm{X}}_{\mathrm{id}}^{\operatorname{cod}} \\
\Delta \overline{\mathrm{X}}_{\mathrm{vem} 4}^{\operatorname{cod}}
\end{array}\right]=\mathrm{T} \Delta \bar{\Theta}
$$

If the new position and angle downstream of VCM4 is along the unperturbed closed orbit, then the bump will remain local. Hence, for open-loop steering of the photon beam, the corrector strengths can be found from $T^{-1}$ with $\Delta \bar{x}_{\mathrm{vem} 4}^{\infty \mathrm{d}}=(0,0)$. Although the position and angle of the electron beam at VCM4 was chosen, any position outside the bump would suffice. Also, equation (3) can easily be generalized to account for any coupling between the vertical and horizontal dimensions.

\section{FEEDBACK CONTROL SYSTEM MODEL}

Equation (3) provides the corrector strengths for static beam steering. However, due to modeling uncertainties and exogenous disturbances, a dynamic system is necessary. In order to "close the loop" on the photon beam position, an accurate model for entire control system needs to be developed. Shown in Fig. 2, the feedback control algorithm consists of a controller (to be developed in Sections IV and V), the beam dynamics compensation gain $\left(T^{-1}\right)$, and vacuum chamber compensation filters. The transfer function for the accelerator (i.e. power supply inputs to photon beam position monitors) consist of the superposition of four decoupled corrector magnets systems. The dynamics of this system is dominated by the eddy currents effects of the aluminum vacuum chamber. The cutoff frequencies for the vacuum chamber at the correctors are $(58 \mathrm{~Hz}, 195 \mathrm{~Hz}, 250 \mathrm{~Hz}, 58 \mathrm{~Hz})$ vertically and $(3 \mathrm{~Hz}, 55 \mathrm{~Hz}, 66 \mathrm{~Hz}, 3 \mathrm{~Hz})$ horizontally. The power supply and magnet bandwidths are approximately 500 $\mathrm{Hz}$ and $1000 \mathrm{~Hz}$, respectively.

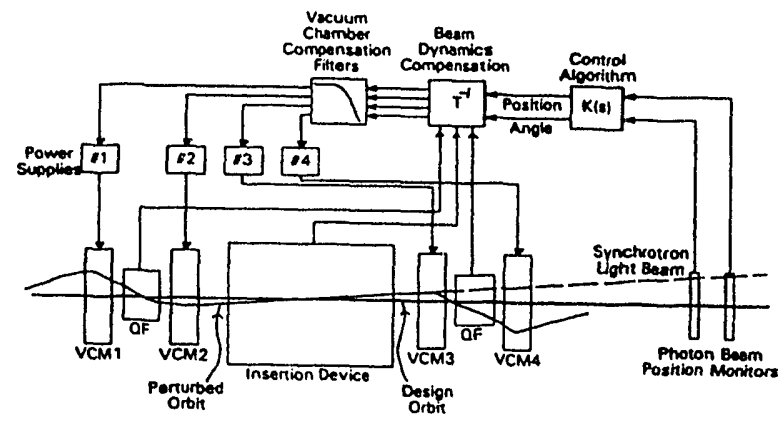

Fig. 2. Local Bump Control System Model

The power supply range is \pm 100 amps vertically and \pm 40 amps horizontally (accurate to 1 part in 10,000), which corresponds to a \pm 3.2 milliradian change in the electron beam at $1.5 \mathrm{GeV}$. The photon beam position monitors
(PBPM), located 8 and 12 meters from the insertion device center, are accurate to \pm 1 micron.

The inherent difficulty in designing a feedback control law for this system is due to the fact that only two position monitors are available when one is trying to control four decoupled corrector magnet systems. The fact that the states in each of the correctors are decoupled makes the plant unobservable. Lack of observability extremely limits feedback control design. As done in [5] and [6], essentially one has to take an open-loop control approach, then feedback on two PBPM signals with the assumption that the zero leakage condition is met. The problem with this approach is that closed-loop control only provide robustness to modeling uncertainties for the photon beam steering, and no robustness on the leakage condition. Hence, the controller performance and stability will be extremely sensitive to modeling errors.

The remainder of this paper will concentrate on the controller design, $K(\mathrm{~s})$. The design example will be a four magnet local bump in the vertical dimension with a closedloop bandwidth of $100 \mathrm{~Hz}$. For simplicity, only an analog control design will be considered, however, the actual implementation will be digital for flexibility.

\section{CLASSICAL CONTROL DESIGN}

The fundamental goal of a feedback control system is to track a given input command given actuator limitations, uncertainties in the dynamics model, sensor noise, and exogenous disturbances. A generic feedback control structure is shown in. Fig. 3. The power supplies, magnets, and accelerator physics models are lumped into block $G(s)$ (the plant), with modeling uncertainties, $\Delta(\mathrm{s})$. The disturbances are external inputs not accounted for in the design model, e.g. vibration, power supply ripple, cross-talk errors, and temperature drift.

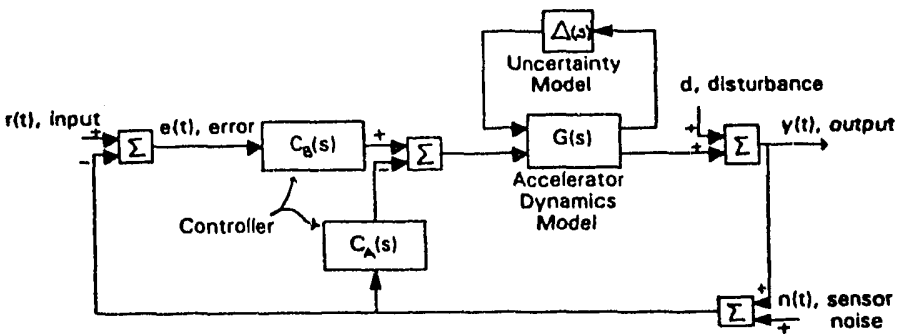

Fig. 3. Feedback Control System Structure

If $C_{A}=0$, then the equations of interest are the following.

$$
\begin{aligned}
& \mathrm{y}=\mathrm{GC}_{\mathrm{B}}\left(\mathrm{I}+\mathrm{GC}_{\mathrm{B}}\right)^{-1}(\mathrm{r}-\eta)+\left(\mathrm{I}+\mathrm{GC}_{\mathrm{B}}\right)^{-1} \mathrm{~d} \\
& \tilde{\mathrm{e}} \equiv \mathrm{r}-\mathrm{y}=\left(\mathrm{I}+\mathrm{GC}_{\mathrm{B}}\right)^{-1}(\mathrm{r}-\mathrm{d})+\mathrm{GC}_{\mathrm{B}}\left(\mathrm{I}+\mathrm{GC}_{\mathrm{B}}\right)^{-1} \eta
\end{aligned}
$$

The sensitivity function is defined as the $d$ to $y$ transfer function, $S=\left(I+G C_{B}\right)^{-1}$, and the complementary sensitivity function is the $r$ to $y$ transfer function, $T=G C_{B}(I$ $+\left(\mathrm{GC}_{\mathrm{B}}\right)^{-1}$. The fundamental trade-off in classical control is that $|\mathrm{T}+\mathrm{S}|=\mathrm{I}$ over all frequencies. $|\mathrm{S}|$ "small" implies good tracking performance, however, it forces $|T|$ close to unity. Since $G$ naturally rolls off at high frequency, this 
would force $\mathrm{K}$ to increase which is destabilizing since the knowledge of the plant dynanucs at high frequency is poor. This is discussed in detail in [2] and [4]. Classical PID control and lead-lag compensation can be used to adjust the trade-off. For the vertical local bump the following PI control law provides the necessary performance, $\tau=.0016$ seconds.

$$
C_{B}(s)=\frac{1.4[s+650]}{s}, C_{A}(s)=0
$$

\section{A TWO DEGREE-OF-FREEDOM CONTROL DESIGN}

By using two "handles", $C_{A}$ and $C_{B}$, to adjust the control signal, [1], one can remove the fundamental trade-off in Section IV. Define,

$$
\begin{aligned}
& C_{\mathrm{A}}=\tilde{G}^{-1} \mathrm{Q}(\mathrm{I}-\mathrm{Q})^{-1} \\
& \mathrm{C}_{\mathrm{B}}=\mathrm{K}(\mathrm{I}-\mathrm{Q})^{-1}
\end{aligned}
$$

where $\tilde{G}$ is the estimate of $G$. There are two cases of interest. If $\tilde{G}=G$, then the output and error transfer functions are the following.

$$
\mathrm{y}=(\mathrm{I}+\mathrm{GK})^{-1} \mathrm{GKr}+(\mathrm{I}-\mathrm{Q})(\mathrm{I}+\mathrm{GK})^{-1} \mathrm{~d}-(\mathrm{I}+\mathrm{GK})^{-1}(\mathrm{Q}+\mathrm{GK}) \eta
$$

$\tilde{\mathbf{e}}=(\mathrm{I}+\mathrm{GK})^{-1} \mathrm{r}-(\mathrm{I}-\mathrm{Q})(\mathrm{I}+\mathrm{GK})^{-1} \mathrm{~d}+(\mathrm{I}+\mathrm{GK})^{-1}(\mathrm{Q}+\mathrm{GK}) \eta$

Therefore, if the estimate of the plant is accurate, then the $Q$ function acts as a scaling term to adjust the influence of the two error sources on the output and error. In the limit, if $Q=I$, then

$$
\begin{aligned}
& y=\left(G \tilde{G}^{-1}+G K\right)^{-1} G K r-\eta \\
& \tilde{e}=\left(G \tilde{G}^{-1}+G K\right)^{-1} G \tilde{G}^{-1} r+\eta
\end{aligned}
$$

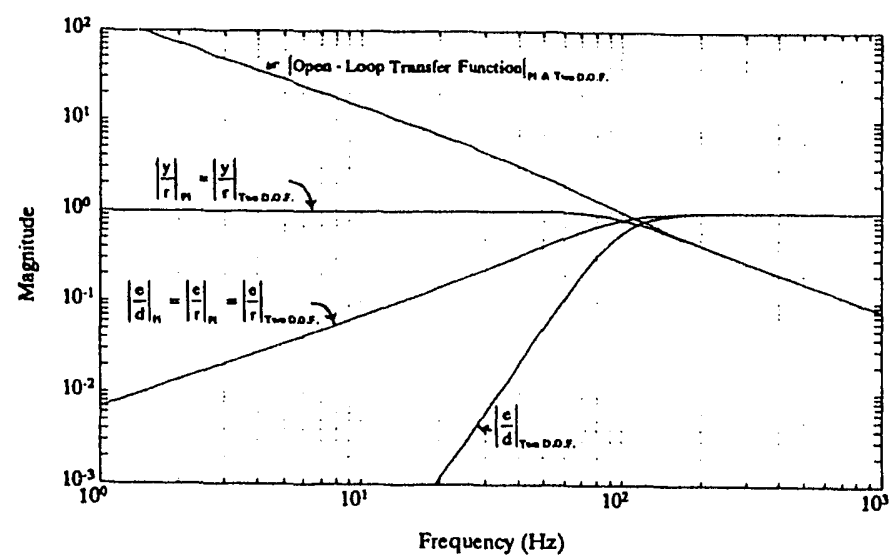

Fig. 4. Disturbance to Error and Reference Input to Error Transfer Functions.

Notice that the disturbance has been totally eliminated -even when $\tilde{\mathbf{G}} \neq \mathbf{G}$. Given these facts, the design goal is to choose $Q \approx I$ inside the system bandwidth. The following design example will use a third order Butterworth filter with the cutoff frequency set at the system bandwidth, $100 \mathrm{~Hz}$. K will be the same PI controller as in (6).
As shown in Fig. 4, the two degree-of-freedom approach has superior disturbance rejection over the simple PI controller - $80 \mathrm{~dB}$ per decade verses $20 \mathrm{~dB}$ per decade. However, the reference input to error and closed-loop transfer functions are identical. As discussed in [1], the two degree-of-freedom controller can be formulated as a disturbance observer-based system. By estimating and canceling the disturbance, one will not only reduce environmental error sources but also the negative effects of cross-talk between the local bumps.

\section{CONCLUSION}

The development of an accurate closed orbit model for the ALS is crucial for the success of the photon beam steering feedback system. The insertion devices will cause relatively large field perturbations when scanned. Since the quadrupole magnets will be adjusted to help compensation for the field changes, the local bump algorithm must continually update the lattice model in order to compute the proper dipole kicks, equation (3). The effects of field variations for the worst case (i.e. ten insertion devices) is currently being studied.

To minimize the effects of cross-talk due to modeling errors as well as environment error sources, a two degree-offreedom control algorithm has been developed. This approach has superior performance over the classical PI controller. It has the added advantage that one can set the system bandwidth independent of the disturbance rejection "bandwidth". The only cost of these benefits is a more demanding computer throughput.

\section{ACKNOWLEDGMENTS}

The authors would like to thank S. Chattopadhyay, the Center for Beam Physics, and H. Lancaster for their continued support and encouragement during this sludy.

\section{REFERENCES}

[1] T.Umeno, Y. Hori, IEEE Trans. Industrial Electronics, Vol. 38, No. 5, Oct. 1991.

[2] Doyle, G. Stein, IEEE Trans. Automatic Control, Vol AC-26, No. 1, Feb. 1981.

[3] C. Chen, "Linear System Theory and Design," 1984.

[4] G.F. Franklin, J.D. Powell, A. Emami-Naeini, "Feedback Control of Dynamic Systems," 1991.

[5] R.J. Nawrocky, J.W. Bittner, Li Ma, H.M. Rarback, D.P. Siddons, and L.H. Yu, Nucl. Instr. and Meth., A266, pp.164-171, corrected Oct. 10, 1988.

[6] R.O. Hettel, Nuc. Inst. \& Methods in Phys. Res., A266, pp. 155-163, 1988.

[7] E.D. Courant and H. S. Snyder, Ann of Phy. 3, 1958.

[8] "1-2 GeV Synchrotron Radiation Source, Conceptual Design Report," PUB-5172 Rev., Lawrence Berkeley Laboratory, 1986.

[9] J. Bengtsson, E. Forest, H. Nishimura, unpublished. [10]MATLAB Reference Guide. 

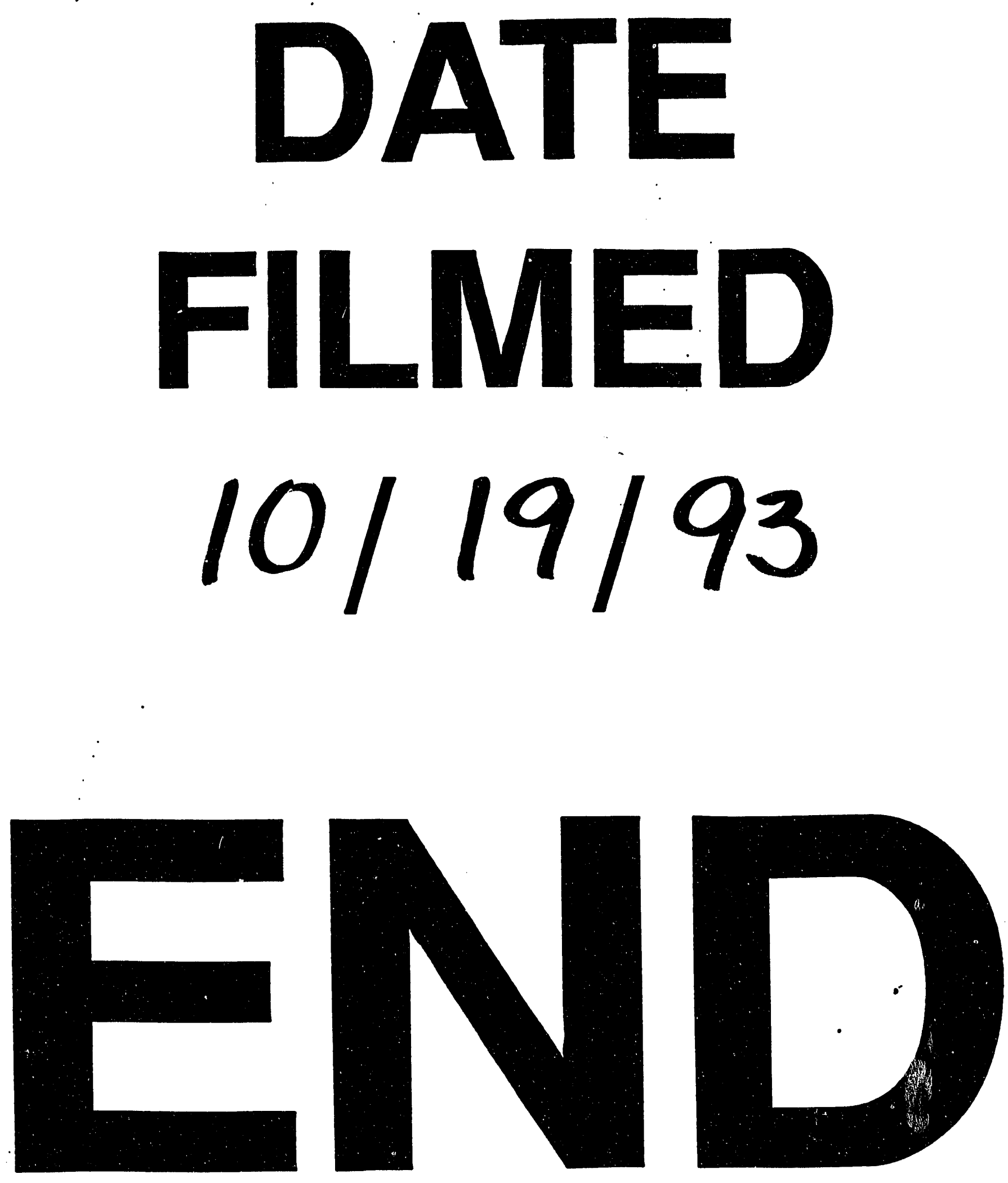
